\section{Magnitude da depressão pós-parto no Brasil: uma revisão sistemática}

\section{The extent of post-partum depression in Brazil: a systematic review}

Gustavo Lobato 1

Claudia L. Moraes 2

Michael E. Reichenheim 3
${ }^{1}$ Instituto Fernandes Figueira. Fundação Oswaldo Cruz. Av. Rui Barbosa, 716, $3^{\circ}$ andar - Maternidade. Flamengo. Rio de Janeiro, RJ, Brasil. CEP: 22.250-020.

E-mail: lobato@iff.fiocruz.br

2,3 Departamento de Epidemiologia. Instituto de Medicina Social. Universidade do Estado do Rio de Janeiro. Rio de Janeiro, RJ, Brasil.

\section{Resumo}

Objetivos: realizar uma revisão sistemática dos estudos sobre a magnitude da depressão pós-parto (DPP) no Brasil.

Métodos: a busca e seleção da literatura baseouse em artigos publicados em periódicos nacionais e internacionais, nas bases de dados eletrônicas Lilacs, SciELO e Medline.

Resultados: foram selecionados 14 estudos, sendo que 13 deles reportavam a prevalência de DPP e apenas um estudo de seguimento com limitada casuistica $(n=21)$ trazia estimativa da incidência do agravo (42,8\%). A grande heterogeneidade em relação à população de estudo, método diagnóstico utilizado $e$ período pós-parto focalizado dificultou a obtenção de uma estimativa agregada da prevalência de DPP no Brasil. Contudo, estudos conduzidos em unidades básicas de saúde, no âmbito da Estratégia de Saúde da Família ou em populações carentes apontaram uma prevalência entre 30 e $40 \%$ de DPP, enquanto pesquisas que incluíram amostras de base populacional e populações de unidades hospitalares terciárias revelaram uma prevalência de cerca de $20 \%$.

Conclusões: embora novos estudos sejam necessários para melhor caracterizar as peculiaridades que envolvem a magnitude da DPP no Brasil, as evidências disponíveis justificam uma atenção prioritária para os agravos à saúde mental materna no âmbito da saúde pública no país.
Palavras-chave Depressão
pós-parto, 


\section{Introdução}

Os agravos à saúde mental são responsáveis por uma significante parcela da morbidade e mortalidade em todo o mundo. Nesse contexto, os transtornos depressivos unipolares assumem a quarta posição dentre os principais determinantes de carga global de doenças, e nos próximos dez anos a depressão pode se tornar a segunda maior causa de doença. O impacto negativo da síndrome depressiva na saúde humana é ainda mais relevante entre as mulheres. Estas apresentam um risco duas vezes maior que os homens de desenvolver depressão, sendo esta diferença ainda mais pronunciada na fase da vida em que elas se vêem responsáveis pelo cuidado de sua prole. 1

O período pós-natal tem sido apontado como uma passagem da vida feminina em que os transtornos mentais são particularmente frequentes. Os riscos inerentes ao sexo feminino encontram-se aqui somados às grandes mudanças impostas pela chegada de um filho ao núcleo familiar, com novas e crescentes responsabilidades, medos e interrogações, além das mudanças físicas e hormonais impostas pela gestação, parto e puerpério. Transformações da família contemporânea, individualismo, precário nível de informação em saúde, pobreza e desagregação social completam o panorama desfavorável.1

Assim, a depressão pós-parto (DPP) é uma condição há muito reconhecida como importante causa de morbidade materna, com grande relevância no âmbito da saúde pública.1,2 Além das sérias conseqüências para sua própria saúde, as síndromes depressivas que acometem mulheres nos primeiros meses após o parto afetam diretamente toda a família. Os cônjuges de mulheres com DPP parecem também mais susceptíveis a desenvolver quadros clínicos de depressão, favorecendo o aparecimento ou agravamento dos conflitos conjugais, e seus filhos mostram-se mais propensos a atraso no desenvolvimento cognitivo e social, distúrbios do sono, doenças diarréicas, distúrbios nutricionais e atraso no crescimento. 1

Apesar desse substancial corpo de evidências clínicas e epidemiológicas que verificam a importância da DPP na saúde materno-infantil, uma avaliação mais minuciosa da literatura revela que as estimativas acerca da magnitude da DPP têm sido questionadas por parte da comunidade científica. Embora diversos pesquisadores sugiram prevalências gerais entre 10 e $15 \%$ em países desenvolvidos, podendo alcançar $40 \%$ em países em desenvolvimento, 1 esses dados devem ser vistos cautelosamente, visto que outros estudos têm apontado números maiores ou menores. ${ }^{3,4}$ Nesse contexto, há de se considerar que diversos fatores culturais, étnicos, socioeconômicos e biológicos parecem contribuir tanto para a gênese como para a manutenção dos quadros de DPP em uma dada população. Estimativas discrepantes podem também ser atribuídas ao emprego de estratégias metodológicas variadas, tais como diferentes desenhos de estudo e diversos instrumentos para o rastreamento ou diagnóstico da DPP. Além disso, a frequência e a severidade dos quadros de DPP podem diferir entre estudos de base populacional e pesquisas realizadas em serviços de saúde.

Embora se reconheça que estimar a frequência do problema e identificar grupos mais vulneráveis sejam os primeiros passos para a elaboração de estratégias efetivas visando a prevenção, diagnóstico precoce e tratamento da DPP, no Brasil, a literatura sobre o tema ainda parece insuficiente. Visando identificar potenciais lacunas no conhecimento sobre a frequência da DPP no Brasil, esta pesquisa teve como objetivo realizar uma revisão dos estudos sobre a magnitude da depressão pós-parto realizados no país até a presente data. Além de apresentar as estimativas oriundas de cada estudo, pretende-se discutir estes dados à luz das diferenças metodológicas e populacionais existentes entre eles, 5 e assim, oferecer ao leitor um panorama do problema em nosso meio.

\section{Métodos}

O levantamento bibliográfico que subjaz a concepção e redação deste estudo foi baseado em uma revisão sistemática das publicações em periódicos nacionais e internacionais, até a data-limite de 16 de agosto de 2010. Nas bases de dados Lilacs (Literatura Latino-Americana e do Caribe em Ciências da Saúde) e SciELO (Scientific Electronic Library Online) foram utilizados os termos "postpartum depression" e "postnatal depression", presentes no título ou no resumo dos artigos, conforme Descritores em Ciências da Saúde (DeCS). Na base de dados Medline (Medical Literature Analysis and Retrieval System Online), conforme sugerido no MeSH (Medical Subject Headings), foram utilizadas as expressões "postpartum depression" e "postnatal depression", ambas associadas ao termo "Brazil", os quais deveriam estar presentes no título ou no resumo - postpartum[Title/Abstract]) AND depression[Title/Abstract]) AND Brazil [Title/Abstract]; ou postnatal [Title/Abstract]) AND depression [Title/Abstract]) AND Brazil [Title/Abstract]. 
Visando evitar a perda de quaisquer publicações potencialmente elegíveis, os estudos foram inicialmente analisados através de seus títulos e resumos. Foram selecionadas para a avaliação de sua íntegra as publicações cujos sumários sugerissem a presença, no texto principal, de resultados originais sobre a prevalência ou incidência de DPP na população de estudo. Não foram aplicados quaisquer critérios de exclusão baseados no escopo temporal das investigações, idioma e ano de publicação, tamanhos amostrais, delineamentos dos estudos ou instrumentos diagnósticos utilizados para a caracterização da DPP. Nessa etapa foram excluídos apenas artigos que não eram disponíveis em formato digital nas bibliotecas nacionais. Também foi realizada uma busca secundária na lista bibliográfica dos artigos inicialmente avaliados, com o objetivo de identificar outras importantes referências não captadas pela busca inicial.

Além dos dados sobre a magnitude de DPP, foram compiladas informações sobre aspectos metodológicos que pudessem ter alguma influência nos estimativas de prevalência (ou incidência) de casos suspeitos ou diagnosticados de DPP, especialmente o delineamento do estudo, perfil sociodemográfico da população avaliada, período pós-parto abarcado e instrumento utilizado para a identificação dos casos. Todo o processo de busca nas bases de dados eletrônicas, seleção dos estudos, leitura dos artigos e compilação das informações foi realizada pelo autor principal (GL) do estudo. Visando maior confiabilidade do estudo, esse processo de busca e seleção dos artigos foi replicado pelo mesmo observador (GL) de maneira cega e independente, não sendo identificadas discrepâncias quanto à seleção final dos artigos.

\section{Resultados}

Conforme apresentado na Figura 1, a busca eletrônica inicial identificou 186 estudos nas bases Lilacs, SciELO e Medline. Porém, apenas 15 artigos originais apresentavam títulos ou resumos que sugerissem a presença, no texto completo, de dados sobre a frequência da DPP. Dois desses estudos foram posteriormente excluídos, um por não estar disponível em formato digital nas bibliotecas nacionais quando da finalização da busca e seleção dos estudos, ${ }^{6}$ e outro por não oferecer estimativas sobre a proporção de casos de DPP em toda a população estudada. ${ }^{7}$ Ainda, uma publicação ${ }^{8}$ foi incluída a partir do escrutínio da lista bibliográfica dos 14 manuscritos identificados a partir da busca eletrônica e lidos na íntegra.
Dentre esses 14 estudos, dez $(71,4 \%)$ artigos se caracterizam como estudos observacionais, transversais, e assim suas estimativas sobre a magnitude da DPP referem-se a casos prevalentes. ${ }^{8-17}$ Três outras publicações $(21,4 \%)$ originaram-se de pesquisas observacionais de seguimento, ${ }^{18-20}$ porém os dados relativos à DPP foram obtidos em um único momento, e então também caracterizam estimativas sobre a prevalência do agravo. Apenas um estudo $(7,1 \%)^{21}$ efetivamente acompanhou as participantes ao longo de um determinado período, com aferições periódicas dos sintomas depressivos pós-natais, assim obtendo uma estimativa da incidência do agravo.

A Tabela 1 sintetiza os principais achados referentes aos estudos selecionados. As estimativas sobre a prevalência da DPP variaram entre 7,2\%17 e $39,4 \%, 14$ muito embora a maior parte das pesquisas detectasse prevalências de DPP entre 15 e $28 \%$ ( $n=9$, $64,3 \%$ ).10,12,13,15,16,18-20,22 A única estimativa da incidência de DPP foi de 42,8\%,21 porém oriunda de estudo com casuística restrita $(n=21)$ e elevado percentual de perdas de seguimento $(27,6 \%)$.

Em relação ao período de publicação, houve um predomínio de estudos publicados nos últimos cinco anos $(n=9,64,3 \%) ; 12-20$ e apenas dois $(14,3 \%)$ são da década de $1990.9,21$

O período pós-natal em que os sintomas depressivos maternos foram avaliados variou entre os estudos selecionados. Embora todos os estudos tenham se restringido aos seis primeiros meses após o parto, a maior parte focalizou algum período compreendido entre 6 e 12 semanas puerperais $(\mathrm{n}=11,78,6 \%$ ).9-12,14-18,20,21 Uma menor parcela das publicações incluiu a avaliação da saúde mental materna no primeiro mês $(\mathrm{n}=7,50 \%), 8,13,14,17-19,21 \mathrm{e}$ apenas cinco focalizaram períodos após o quarto mês pós-natal $(\mathrm{n}=5,35,7 \%) .9,11,14,17,21$

Em relação aos contextos e perfis populacionais dos estudos incluídos na revisão, apenas três $(21,4 \%)^{12,15,18}$ tiveram suas populações baseadas em amostras aleatórias de base populacional, todos realizados no Estado do Rio Grande do Sul. Ainda, três outros estudos $(21,4 \%)$ foram realizados em unidades de atenção básica à saúde ${ }^{14,19}$ ou no âmbito da Estratégia de Saúde da Família (ESF), ${ }^{11}$ enquanto cinco pesquisas $(35,7 \%)$ foram realizadas com mulheres cujos partos ocorreram em hospitais universitários ou unidades de referência para assistência perinatal de alta complexidade. $8,10,13,17,20$

No que tange aos instrumentos de detecção utilizados para o rastreio ou diagnóstico da DPP, oito estudos $(57,1 \%)$ empregaram a Edinburgh Postnatal Depression Scale (EPDS),10,11,13-15,19-21 enquanto 
Fluxograma ilustrativo do processo de busca e seleção dos artigos incluídos na revisão.

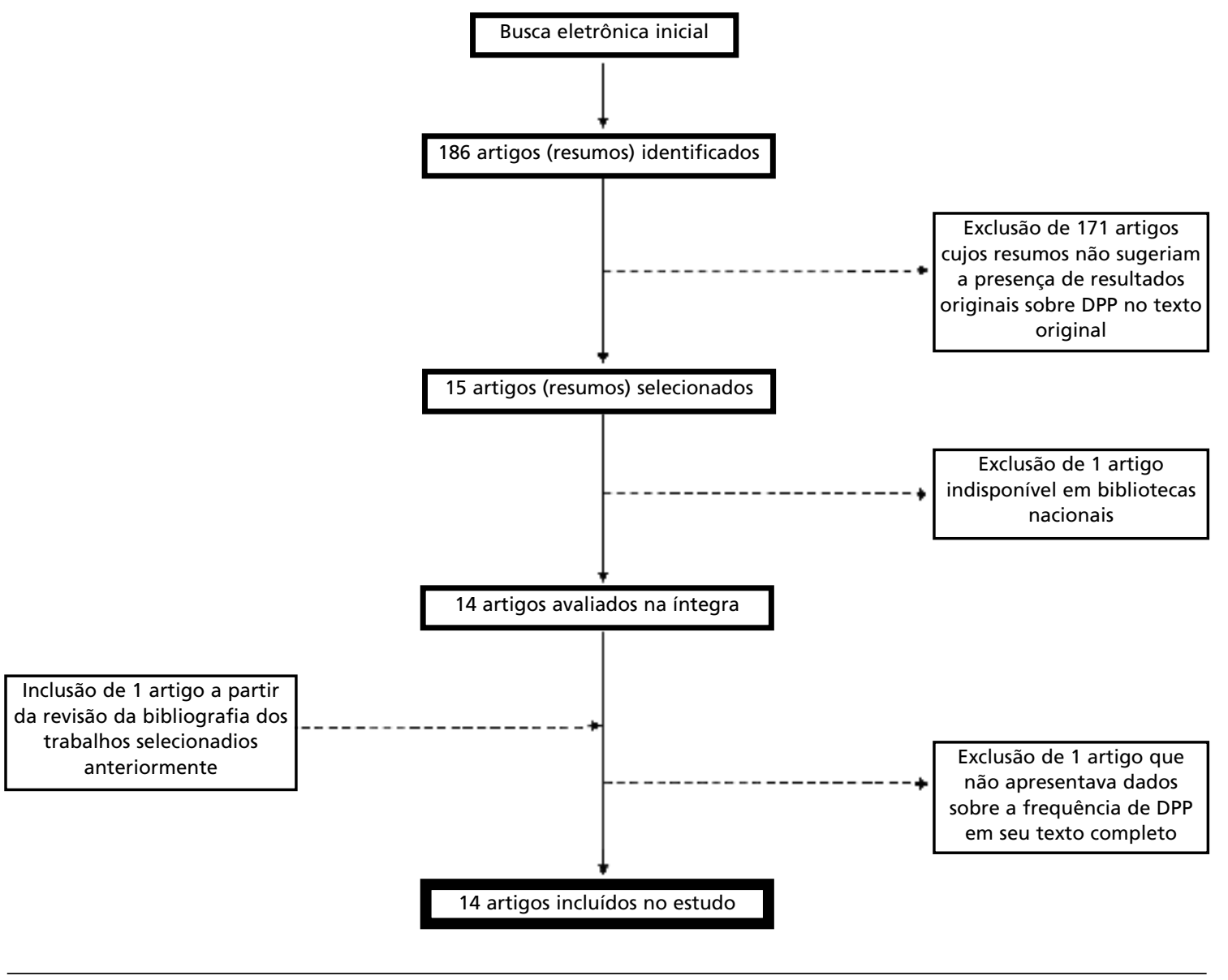

$\mathrm{DPP}=$ depressão pós-parto 


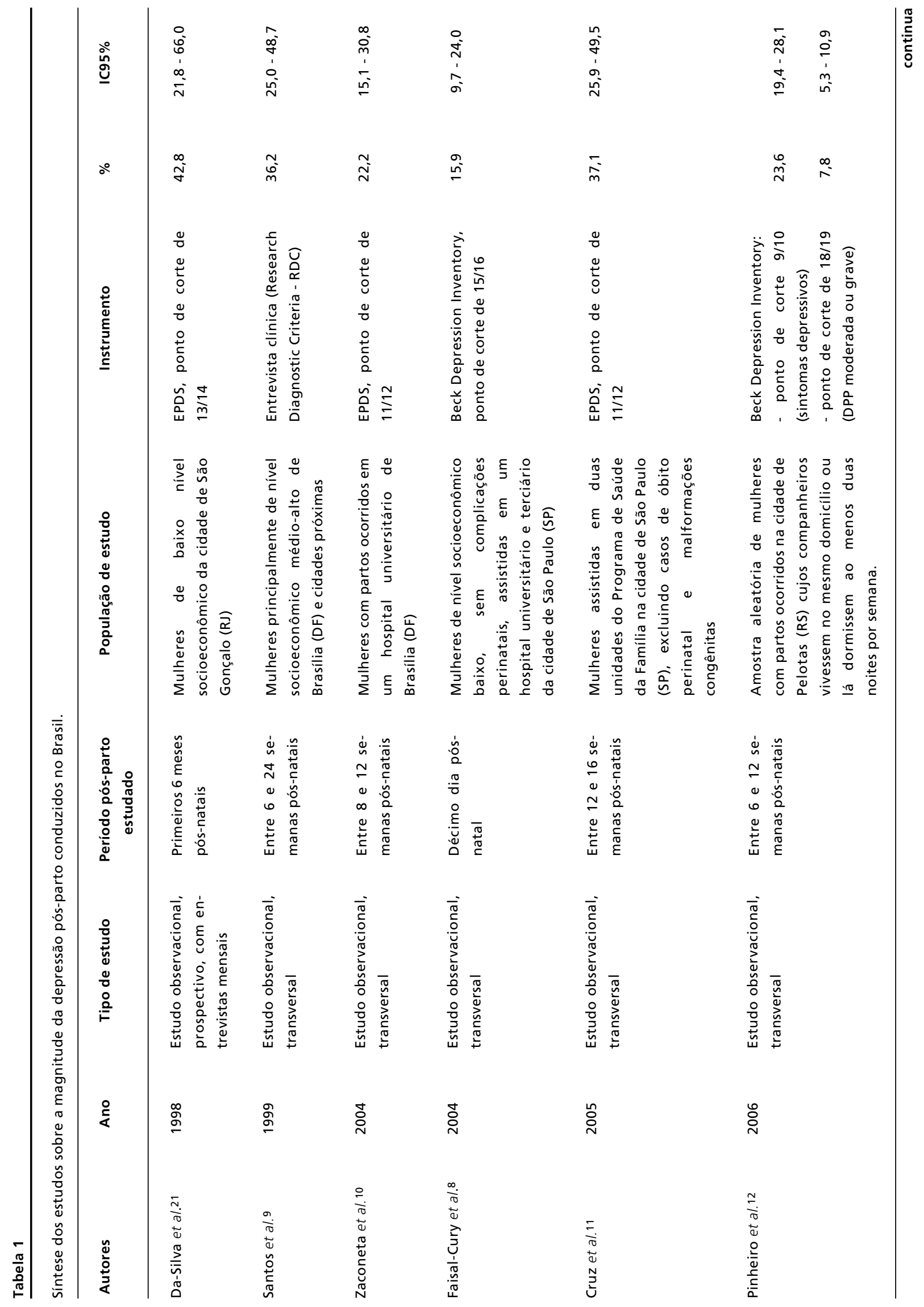




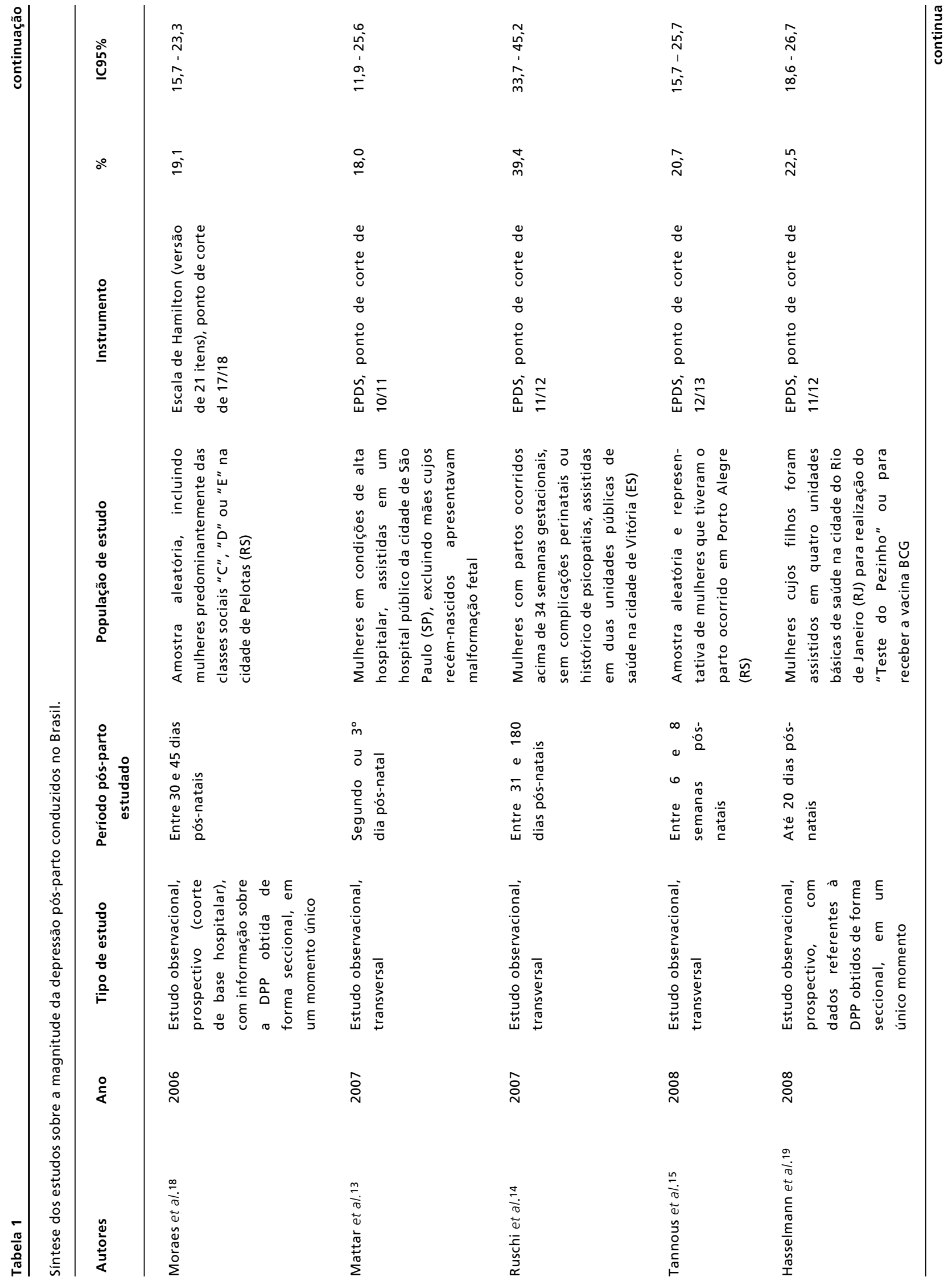




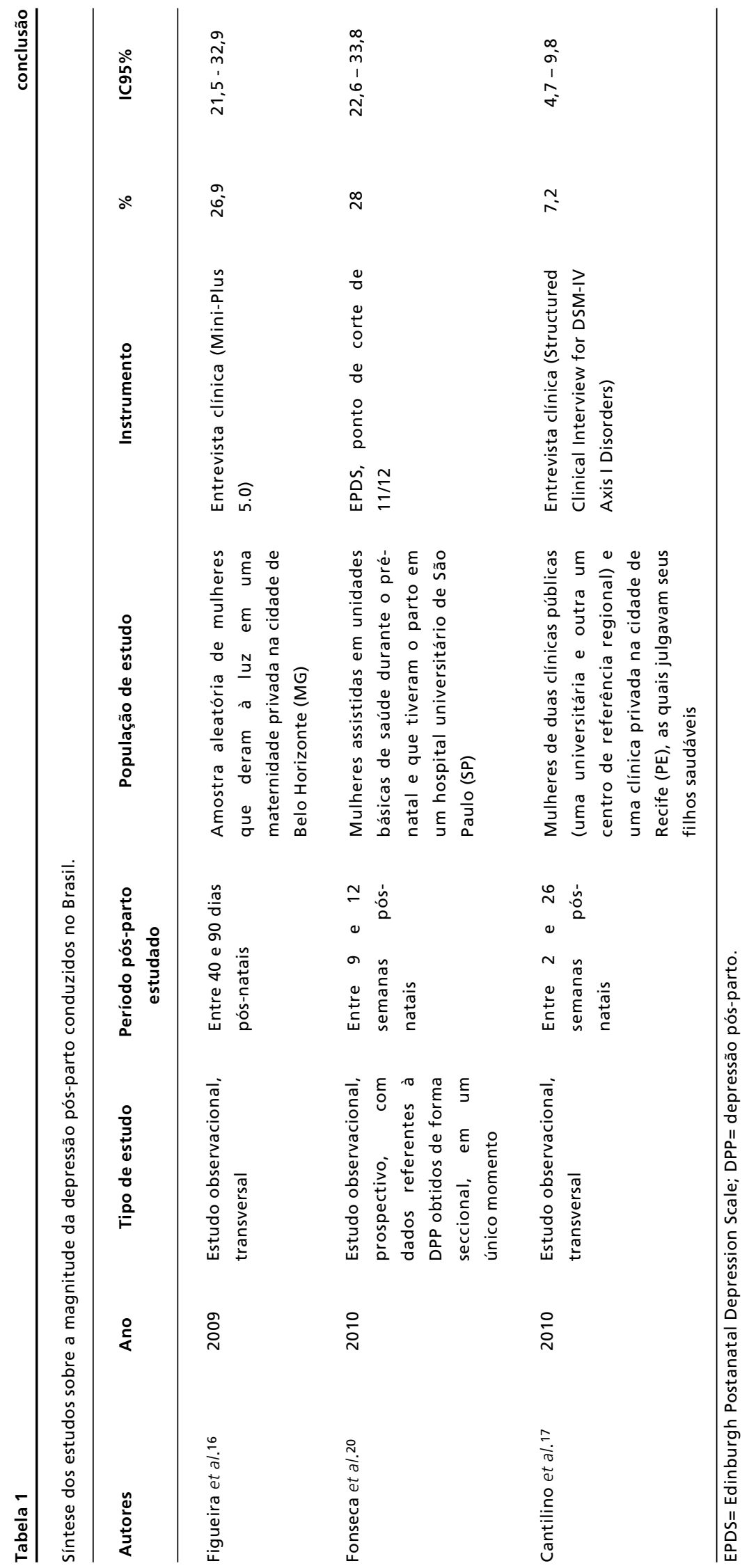


apenas em três publicações $(21,4 \%)$ a entrevista clínica foi utilizada para a caracterização da DPP. $9,16,17$

\section{Discussão}

Os achados deste estudo estão em consonância com os resultados advindos de pesquisas internacionais sobre o tema. A literatura disponível sobre a frequência da depressão pós-parto é baseada em pesquisas com metodologias muito distintas, as quais oferecem resultantes bastante heterogêneos e, consequentemente, dificultam sua compilação em torno de uma estimativa única da magnitude da DPP no Brasil. De qualquer forma, as estimativas sobre a prevalência de sintomas depressivos pós-natais no Brasil não parecem diferir substancialmente quando consideradas pesquisas semelhantes conduzidas em países socioeconomicamente similares, tais como Itália $(38,1 \%)$, Chile $(37,4 \%)$, África do Sul $(36,5 \%)$, Índia $(32,4 \%)$ e Turquia $(29,8 \%) .4$ Nos países ricos, contudo, a DPP parece acometer uma proporção consideravelmente menor de mulheres. 1

Alguns aspectos metodológicos merecem especial atenção quando da interpretação dos resultados dos estudos selecionados. O primeiro deles diz respeito ao tipo de desenho empregado. A maioria das pesquisas disponíveis, sejam elas mundiais 3,4 ou brasileiras, têm um delineamento transversal, com a avaliação da saúde mental materna sendo feita em apenas um momento no tempo. Dessa forma, em geral temos uma estimativa da prevalência da DPP em um determinado período pós-natal, não sendo possível precisar a real incidência do agravo, i.e., o número de casos novos de doença. Tendo em vista que a prevalência de uma doença na população é função não só de sua incidência, mas também da duração média do agravo, em populações onde certas características contribuam para uma lenta recuperação ou mesmo a para a cronicidade dos quadros depressivos as estimativas sobre a prevalência de DPP serão maiores, sem que isso necessariamente signifique um maior risco de adoecimento. Assim, seriam oportunas pesquisas que procurassem discernir se as características socioeconômicas são fatores de risco para a DPP, acarretando uma maior incidência entre grupos menos favorecidos, ou se essas características atuam como fatores prognósticos da DPP, contribuindo para uma maior duração da DPP entre populações mais carentes.

Cabe também ressaltar que uma adequada caracterização do tipo de população estudada é fundamental para a interpretação dos achados de um determinado estudo. Neste sentido, o estudo de popu- lações muito específicas ou amostras de conveniência comprometem a generalização dos achados para outros contextos. Deste modo, médias ditas globais de DPP entre 10 e $15 \%$ podem não ter significado no âmbito da saúde materno-infantil brasileira, visto que parecem refletir a frequência da doença entre populações brancas de classe média em países com melhores condições socioeconômicas. ${ }^{1}$ Ainda, estudos realizados com amostras populacionais ou em serviços de saúde podem revelar diferentes magnitude do agravo. Uma análise mais minuciosa dos estudos conduzidos no Brasil mostra que, em linhas gerais, cerca de 30 a $40 \%$ das mulheres atendidas em unidades básicas de saúde, na ESF ou com perfil socioeconômico baixo apresentaram elevados níveis de sintomas depressivos. Em contrapartida, pesquisas de base populacional e pesquisas com enfoque em mulheres assistidas em unidades hospitalares de referência revelaram que aproximadamente $20 \%$ delas apresentavam-se provavelmente deprimidas. De qualquer forma, estudos de base populacional sobre a DPP são ainda escassos e, no Brasil, encontram-se restritos ao Estado do Rio Grande do Sul. Conforme discutido anteriormente, estudos populacionais com maior abrangência nacional são fundamentais para a caracterização da saúde mental materna no país.

As pesquisas sobre DPP também divergem substancialmente em relação ao período pós-natal estudado. Essa disparidade nos remete à opinião de alguns autores de que a DPP ainda carece de uma definição universalmente aceita.23,24 Enquanto a Classificação Estatística Internacional de Doenças e Problemas Relacionados à Saúde - $10^{\mathrm{a}}$ Revisão (CID-10) classifica como transtornos mentais associados com o puerpério aqueles que se iniciam dentro de seis semanas pós-natais, o Diagnostic and Statistical Manual of Mental Disorders - Fourth Edition (DSM-IV), publicado pela Associação Psiquiátrica Americana, define que uma psicopatia pós-parto é aquela que se inicia em até quatro semanas após o parto. Porém, na última década, especialistas de diversas partes do Mundo têm sugerido que essa janela temporal seja estendida para três meses pós-natais. ${ }^{25,26}$ Ainda, há controvérsias sobre o significado dos sintomas depressivos até quinze dias pós-natais, na medida em que podem refletir o quadro de blues (ou disforia) pós-parto e não o diagnóstico clínico de depressão propriamente dito. Nesse sentido, a inclusão na presente revisão de três estudos que focalizaram exclusivamente sintomas depressivos nos primeiros vinte dias após o parto merece atenção, visto que na verdade podem refletir apenas sintomas depressivos e de ansiedade 
leves e autolimitados, que em geral remitem espontaneamente e não chegam a caracterizar um quadro depressivo. Além de uma melhor definição acerca do período a ser utilizado para a definição de um transtorno mental pós-parto, futuros estudos poderiam inclusive focalizar as potenciais diferenças na incidência e prevalência desses agravos ao longo dos primeiros meses pós-parto, o que certamente facilitaria a elaboração, pelo setor saúde, de estratégias e programas mais efetivos no que diz respeito à sua prevenção, diagnóstico precoce e tratamento.

Outro ponto de convergência entre a literatura mundial e a brasileira sobre DPP diz respeito ao predomínio de estudos que fazem uso da EPDS como instrumento de aferição para as síndromes depressivas pós-natais. ${ }^{27}$ A EPDS foi originalmente proposta como um instrumento para a avaliação inicial de quadros de DPP.28 À época de sua concepção, seus idealizadores argumentavam que seria necessário um instrumento específico para se investigar a depressão no período pós-natal, já que os instrumentos disponíveis até então tinham como foco populações gerais e, por isso, punham grande ênfase em sintomas somáticos, os quais podem ser causados pelos processos fisiológicos adaptativos do período pós-parto. No Brasil, os autores responsáveis pelos dois estudos que realizaram a adaptação trans-cultural (ATC) da versão brasileira sugeriram a utilização de diferentes pontos de corte para a identificação da DPP. Santos et al., ${ }^{9}$ em um estudo realizado na cidade de Brasília (DF), identificaram que o ponto de corte $11 / 12$ seria o mais adequado ao contexto brasileiro, com acurácia de $83 \%$. Já em estudo realizado em 2007, na cidade de Pelotas (RS), os autores identificaram que o ponto de corte de $9 / 10$ seria o mais adequado para o rastreio de DPP, enquanto um ponto de corte de 10/11 seria mais apropriado para a identificação de casos de DPP moderada ou grave. ${ }^{7}$

Embora a fácil utilização da EPDS, inclusive por profissional de saúde não-especializado, certamente seja um aspecto positivo seja no âmbito clínico como na pesquisa aplicada, há de se ressaltar que um instrumento diagnóstico simplificado como a EPDS traz limitações quando da inferência sobre a real frequência da DPP, possivelmente superestimando sua ocorrência. Inclusive, a proposta de utilização da EPDS pelos autores da versão original ${ }^{29}$ sugere uma reavaliação da mulher que se apresente com um escore elevado da EPDS cerca de 15 dias após a primeira entrevista. Essa reavaliação poderia ocorrer através da própria EPDS ou de uma entrevista clínica especializada, a qual propiciaria o diagnóstico definitivo de DPP. Nesse sentido, Cantilino et al.17 sugerem que, quando o diagnóstico de DPP é baseado em uma entrevista clínica, sua frequência é sensivelmente inferior e possivelmente mais próxima da real estimativa do agravo.

Algumas limitações metodológicas devem ser consideradas quando da avaliação dos achados desta revisão. No que tange à bibliografia selecionada, foram apenas considerados os estudos publicados na forma de artigos originais e disponíveis em bibliotecas nacionais, não sendo considerados, por exemplo, os achados veiculados em teses ou dissertações. Contudo, esta peculiaridade não parece ter influenciado de forma significativa achados, visto que os estudos mais importantes são publicados no geral em periódicos. Quanto ao processo de seleção e avaliação dos artigos, embora apenas um observador tenha sido responsável pelos procedimentos, sua replicação não gerou nenhuma discrepância.

Em síntese, apesar da expressiva heterogeneidade dos aspectos metodológicos empregados nas diversas pesquisas sobre a magnitude da depressão pós-parto no Brasil, os achados desta revisão sugerem que a prevalência de sintomas depressivos pós-parto no Brasil encontra-se acima da média mundial, e próxima àquela encontrada em países similares do ponto de vista socioeconômico. Esse corpo de evidências, embora não possibilite inferências definitivas sobre diversos aspectos relacionados à DPP no Brasil, certamente justifica que os agravos à saúde mental materna recebam atenção prioritária no âmbito da saúde pública. Além de uma maior visibilidade para essas questões nas consultas obstétricas de revisão pós-parto, uma efetiva integração da assistência materno-infantil mostra-se fundamental. Neste sentido, as consultas pediátricas e de puericultura têm sido reconhecidas como excelentes espaços para o diagnóstico da DPP. 30 Essa estratégia pode, de fato, oferecer muitas vantagens. Tendo em vista a regularidade dessas consultas ao longo do primeiro ano de vida, bem como as diversas visitas para o cumprimento do calendário vacinal, vislumbram-se então muitas oportunidades não somente para a identificação da DPP, mas também para o tratamento e o acompanhamento de sua evolução. Além disso, permitiriam a detecção dos casos com início tardio, após a realização da consulta de revisão pós-parto. Com este propósito, seria auspicioso alargar o âmbito de ação dos pediatras e outros profissionais responsáveis pela assistência materno-infantil em nosso meio, então incluindo a avaliação de aspectos relacionados ao ambiente familiar em sua integralidade. Neste contexto, o Programa Saúde da Família é uma alternativa promissora. 


\section{Agradecimentos}

MER e CLM foram parcialmente apoiados pelo Conselho Nacional de Pesquisa (CNPq), processos $\mathrm{n}^{\circ} 306909 / 2006-5$ e 302851/2008-9, respectivamente.

\section{Referências}

1. WHO, UNFPA. Mental health aspects of women's reproductive health. A global review of the literature. Geneva: WHO Press; 2009.

2. Sit DK, Wisner KL. Identification of postpartum depression. Clin Obstet Gynecol. 2009; 52: 456-68.

3. Gaynes BN, Gavin N, Meltzer-Brody S, Lohr KN, Swinson T, Gartlehner G, Brody S, Miller WC. Perinatal depression: prevalence, screening accuracy, and screening outcomes. Evid Rep Technol Assess (Summ). 2005: 1-8. Available from:

http://www.ahrq.gov/downloads/pub/evidence/pdf/peridepr / peridep.pdf

4. Halbreich U, Karkun S. Cross-cultural and social diversity of prevalence of postpartum depression and depressive symptoms. J Affect Disord. 2006; 91: 97-111.

5. Boyle MH. Guidelines for evaluating prevalence studies. Evid Based Mental Health. 1998; 1: 37-9.

6. Zubaran C, Foresti K, Schumacher MV, Amoretti AL, Muller LC, Thorell MR, White G, Madi JM. Validation of a screening instrument for postpartum depression in Southern Brazil. J Psychosom Obstet Gynaecol. 2009; 30: 244-54.

7. Santos IS, Matijasevich A, Tavares BF, Barros AJ, Botelho IP, Lapolli C, Magalhães PV, Barbosa AP, Barros FC. Validation of the Edinburgh Postnatal Depression Scale (EPDS) in a sample of mothers from the 2004 Pelotas Birth Cohort Study. Cad Saúde Pública. 2007; 23: 2577-88.

8. Faisal-Cury A, Tedesco JJA, Kahhale S, Menezes PR, Zugaib M. Postpartum depression: in relation to life events and patterns of coping. Arch Womens Ment Health. 2004; 7: $123-31$.

9. Santos MFS, Martins FC, Pasquali L. Post-natal depression self-rating scales: Brazilian study. Rev Psiq Clin. 1999; 26: $32-40$.

10. Zaconeta AM, Motta LDC, França PS. Depresión postparto: prevalencia de test de rastreo positivo en puérperas del Hospital Universitario de Brasilia, Brasil. Rev Chil Obstet Ginecol. 2004; 69: 209-13.

11. Cruz EBS, Simões GL, Faisal-Cury A. Rastreamento da depressão pós-parto em mulheres atendidas pelo Programa de Saúde da Família. Rev Bras Ginecol Obstet. 2005; 27 : 181-8.

12. Pinheiro RT, Magalhaes PV, Horta BL, Pinheiro KA, da Silva RA, Pinto RH. Is paternal postpartum depression associated with maternal postpartum depression? Population-based study in Brazil. Acta Psychiatr Scand. 2006; 113: 230-2.

13. Mattar R, Silva EYK, Camano L, Abrahão AR, Colás OR, Neto JA, Lippi UG. A violência doméstica como indicador de risco no rastreamento da depressão pós-parto. Rev Bras Ginecol Obstet. 2007; 29: 470-7.
14. Ruschi GEC, Sun SY, Mattar R, Chambô Filho A, Zandonade E, Lima VJ. Postpartum depression epidemiology in a Brazilian sample. Rev Psiquiatr Rio Gd Sul. 2007; 29: 274-80.

15. Tannous L, Gigante LP, Fuchs SC, Busnello ED. Postnatal depression in Southern Brazil: prevalence and its demographic and socioeconomic determinants. BMC Psychiatry. 2008; 8: 1 .

16. Figueira P, Correa H, Malloy-Diniz L, Romano-Silva MA. Edinburgh Postnatal Depression Scale for screening in the public health system. Rev Saúde Pública. 2009; 43 (Suppl. 1): $79-84$.

17. Cantilino A, Zambaldi CF, Albuquerque TLC, Paes JA, Montenegro ACP, Sougey EB. Depressão pós-parto em Recife - Brasil: prevalência e associação com fatores biossociodemográficos. J Bras Psiquiatr. 2010; 59: 1-9.

18. Moraes IGS, Pinheiro RT, Silva RAS, Horta BL, Sousa PLR, Faria AD. Prevalence of postpartum depression and associated factors. Rev Saúde Pública. 2006; 40: 65-70.

19. Hasselmann MH, Werneck GL, Silva CV. Symptoms of postpartum depression and early interruption of exclusive breastfeeding in the first two months of life. Cad Saúde Pública. 2008; 24 (Suppl. 2): S341-52.

20. Fonseca VRJRM, Silva GA, Otta E. Relação entre depressão pós-parto e disponibilidade emocional materna. Cad Saúde Pública. 2010; 26: 738-46.

21. Da-Silva VA, Moraes-Santos AR, Carvalho MS, Martins ML, Teixeira NA. Prenatal and postnatal depression among low income Brazilian women. Braz J Med Biol Res. 1998; 31: 799-804.

22. Faisal-Cury A, Menezes PM. Depressão pós-parto: o papel do obstetra. Femina. 2005; 33: 135-9.

23. Wisner KL, Moses-Kolko EL, Sit DK. Postpartum depression: a disorder in search of a definition. Arch Women Ment Health. 2010; 13: 37-40.

24. Kornstein SG. Gender issues and DSM-V. Archives of women's mental health. 2010; 13: 11-3.

25. Elliott SA. Report on the Satra Bruk workshop on classification of postnatal mental disorders. Arch Women Ment Health. 2000; 3: 27-33.

26. Wisner KL, Chambers C, Sit DK. Postpartum depression: a major public health problem. Jama. 2006; 296: 2616-8.

27. Gjerdingen DK, Yawn BP. Postpartum depression screening: importance, methods, barriers, and recommendations for practice. J Am Board Fam Med. 2007; 20: 280-

28. Cox JL, Holden JM, Sagovsky R. Detection of postnatal depression. Development of the 10-item Edinburgh Postnatal Depression Scale. Br J Psychiatry. 1987; 150: $782-6$ 
29. Cox J, Holden J. Perinatal Mental Health: a guide to the Edinburgh Postnatal Depression Scale (EPDS). London: Royal College of Psychiatrists, Gaskell; 2003.
30. Chaudron LH, Szilagyi PG, Campbell AT, Mounts KO, McInerny TK. Legal and ethical considerations: risks and benefits of postpartum depression screening at well-child visits. Pediatrics. 2007; 119: 123-8.

Recebido em 17 de janeiro de 2011

Versão final apresentada em 26 de maio de 2011

Aprovado em 8 de agosto de 2011 